\title{
Device for Turning Hemispherical Ends - an Innovative Solution
}

\author{
Romeo CIOARĂ \\ Transilvania University of Brasov, Romania, cioarar@unitbv.ro
}

\begin{abstract}
Large series or mass production is to a great extent a characteristic of present day industrial production in several branches; two relevant examples are consumer goods and the automotive industry. Minimizing production costs and increasing productivity are major objectives of industrial activity and contribute significantly to ensuring marketplace competitiveness. In order to attain these goals machines manufacturing industry often uses customized technologies that deploy specialized or special machines dedicated to the respective production type and/or tools and devices that amplify the performance of machines with a wider destination. Conceiving and developing a more or less specialized device being less cost intensive renders it the preferred approach in many cases. The paper presents and discusses such an example, a device for turning hemispherical ends of various components, particularly of the holding bars of certain motor vehicle head restraints. The originality of the device has been recognised by a patent and several diplomas obtained in national and international inventics fairs.
\end{abstract}

\section{Keywords}

device, spherical surfaces, innovative solution, patent

\section{Introduction}

Minimizing production costs and increasing productivity are major objectives of not only of industrial activity, and most often are decisive for securing marketplace competitiveness. Concern in this direction is general and manifests in multiple ways depending on the specifics of various industries and on the volume of production.

In particular, in machines manufacturing industry numerous examples of large series or mass manufactured products can be identified, often characterized by specific surfaces or other technical requirements. In order to maximize productivity and minimize costs often customized technologies are used that deploy either specialized or special machines dedicated to the respective type of production or tools and devices that amplify the performance of machines with a wider destination, or both.

Conceiving and using customized tools, specific for a certain machining procedure is a relatively frequent decision, entails a relatively small investment and given a sufficiently large manufacturing series, will contribute to increasing productivity, even if not spectacularly so.

In many cases the preferred approach is to deploy a more or less specialized device to equip available machine-tools often of an elevated degree of universality. While such a device is costlier than a specific tool, the increase in productivity consequent to its deployment is significant. Often one or more adequate tools are required.

Specialized or special machine-tools, most often semi-automatic or completely automatic are generally expensive, typically require more or less dedicated tools and at times even specific devices, but their deployment has beneficial economic effects: a remarkable increase of productivity and a most attractive unit cost of the machined component, at least for sufficiently large fabrication series.

The automotive industry offers many opportunities for the deployment of and often requests customized technologies of the type mentioned above. All three technological variants described above were taken into consideration by the research conducted by a team of authors towards increasing productivity and ensuring the confidence of repeatability in the machining of motor vehicle head restraint holding bar hemispherical ends. The conducted research yielded as the most performant approach the conceiving of a highly specific device that allows the machining of the head restraint holding bar hemispherical ends subsequently to the bending of the bar, the machining motions being carried out by the device. Also explored was the possibility of using two identical devices in parallel for 
the simultaneous machining of both head restraints holding rod ends.

\section{Prior Art}

Apart from various specific roller bearing elements, components with spherical surfaces are encountered rather infrequently in machines manufacturing. As clamping the respective components is imperative and possibly also rotating these, their spherical surfaces are always of spherical calotte or segment type, never whole spheres. The discussed components are machined by turning or milling.

Turning of spherical surfaces can be achieved by:

- copying, by means of profiled tools. The feed of the tool can be radial, axial or even "tilted" (when the direction of feed is neither perpendicular nor parallel to the rotation axis of the part);

- kinematic generation of the circle arc generatrix, where by means of an adequate device (example in figure 1) the tool carries out a circular feed motion by a vertical axis that includes the centre of the spherical surface;

- copying following a template or by means of NC lathes, in both cases with simultaneously active longitudinal and radial feed.

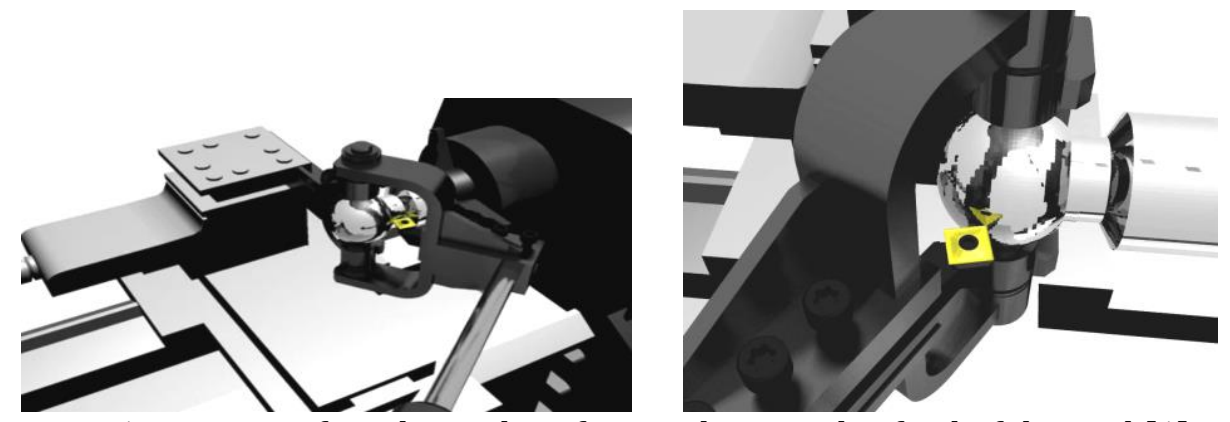

Fig. 1. Turning of a spherical surface with a circular feed of the tool [1]

A particular case of spherical surface turning is put forward in patent US 3142117 [2] using a very specific tubular tool with a materialised generatrix.
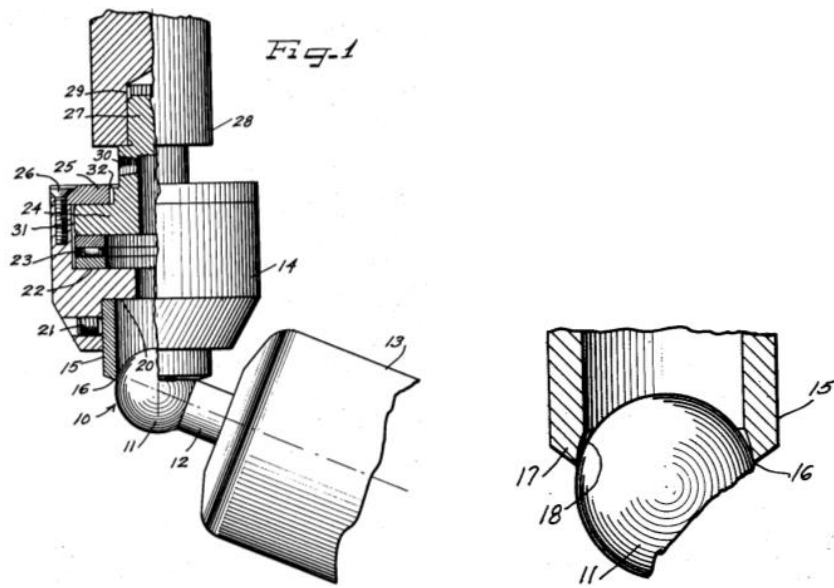

Fig. 2. Turning of a spherical calotte with a tubular tool with a materialised generatrix [2]

Milling of spherical surfaces can be achieved efficiently by:

- copying, using profiled milling cutters. Two possibilities can be identified:

a) both the directrix and the generatrix are copied, both materialised on the tool. Ball end mills can be used (figure 3) that are generally used to machine complex spatial surfaces if the tool profile matches the geometry of the spherical surface to be obtained, or custom design profiled tools are used like the one in figure 4; 

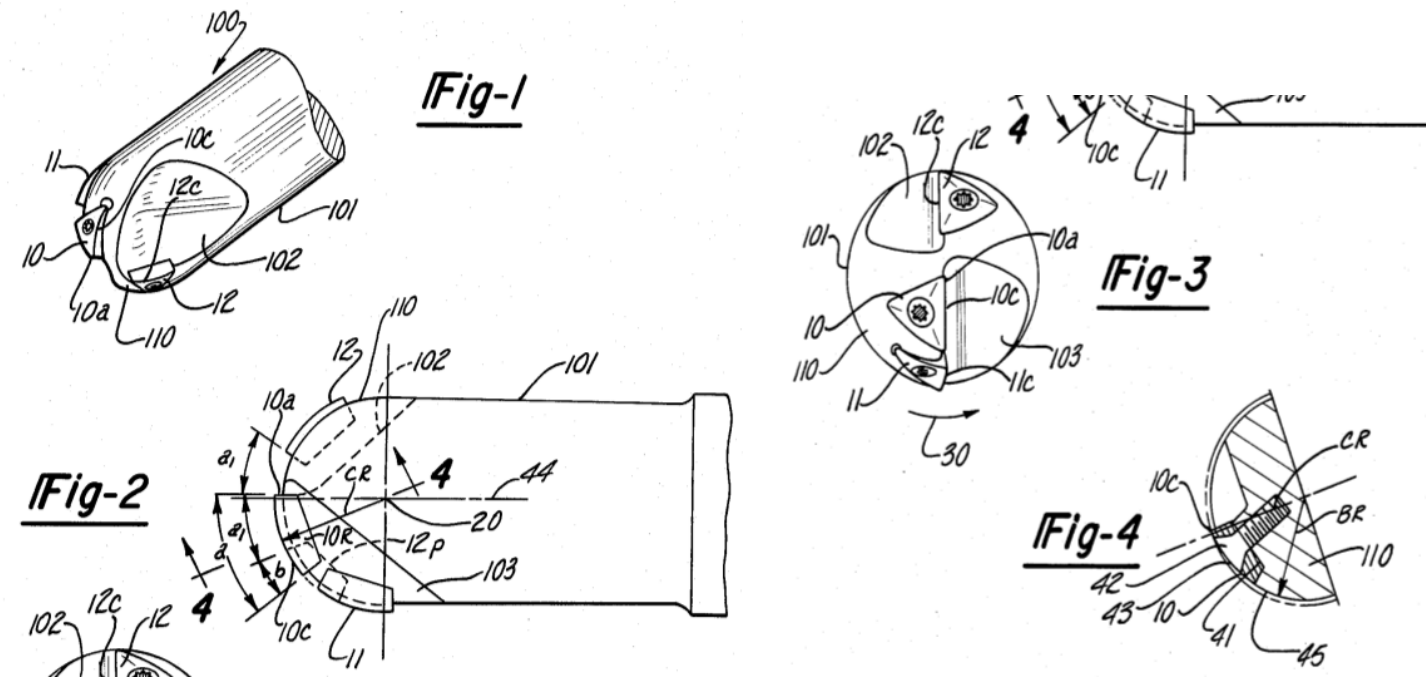

Fig. 3. Ball end mill - a patented solution [3]
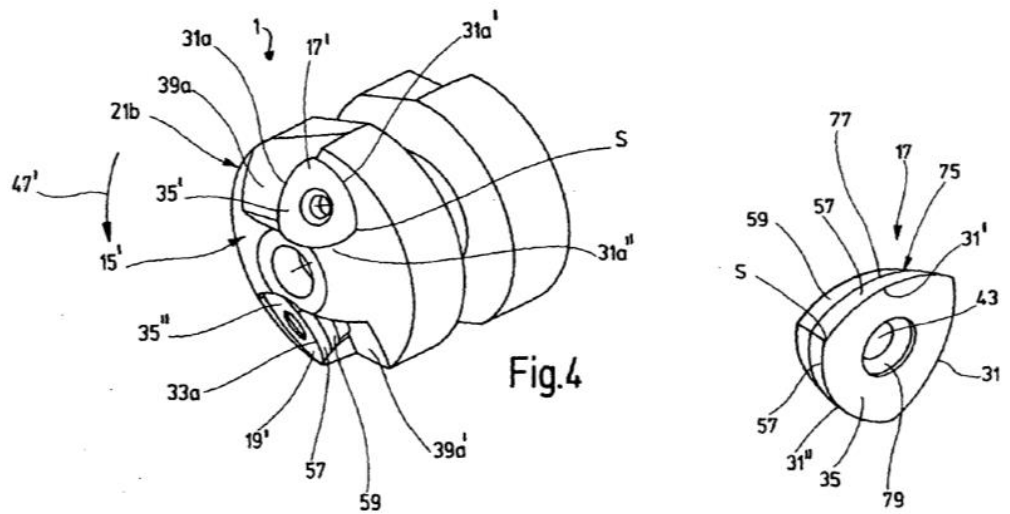

Fig. 4. Special tool for milling concave spherical surfaces - a patented solution [4]

b) only the tool generatrix is copied (typically a profiled disk mill), in which case the machined part has to carry out a circular feed motion;

- kinematic generation by means of end mills, face mills or insert face milling cutters, as exemplified in figures 5 and 6.
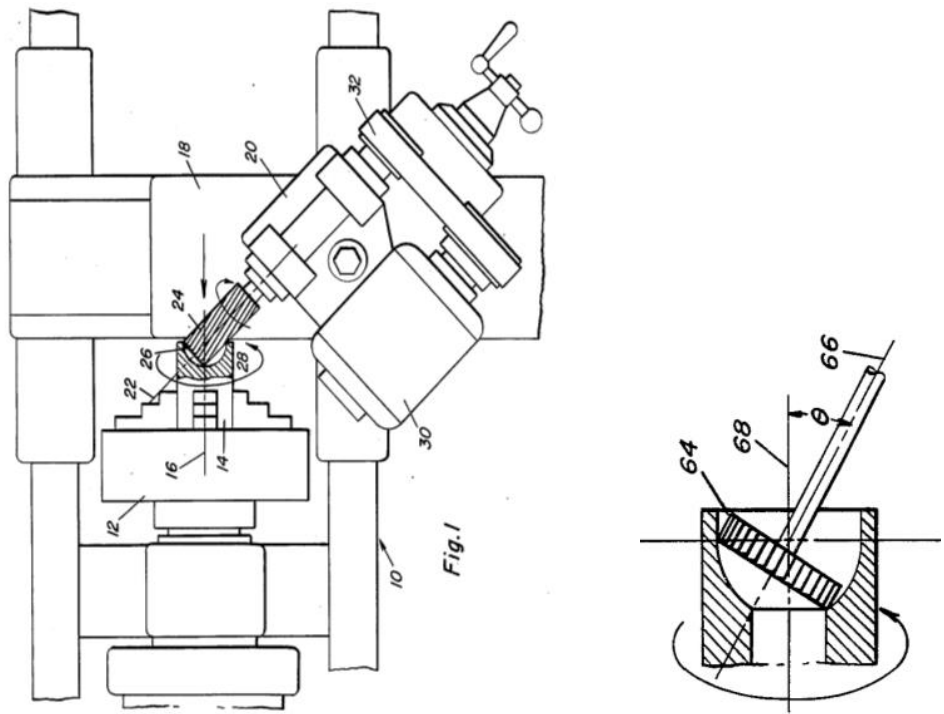

Fig. 5. Schematic of spherical cavity machining by milling with end or face mills, according to [5] 

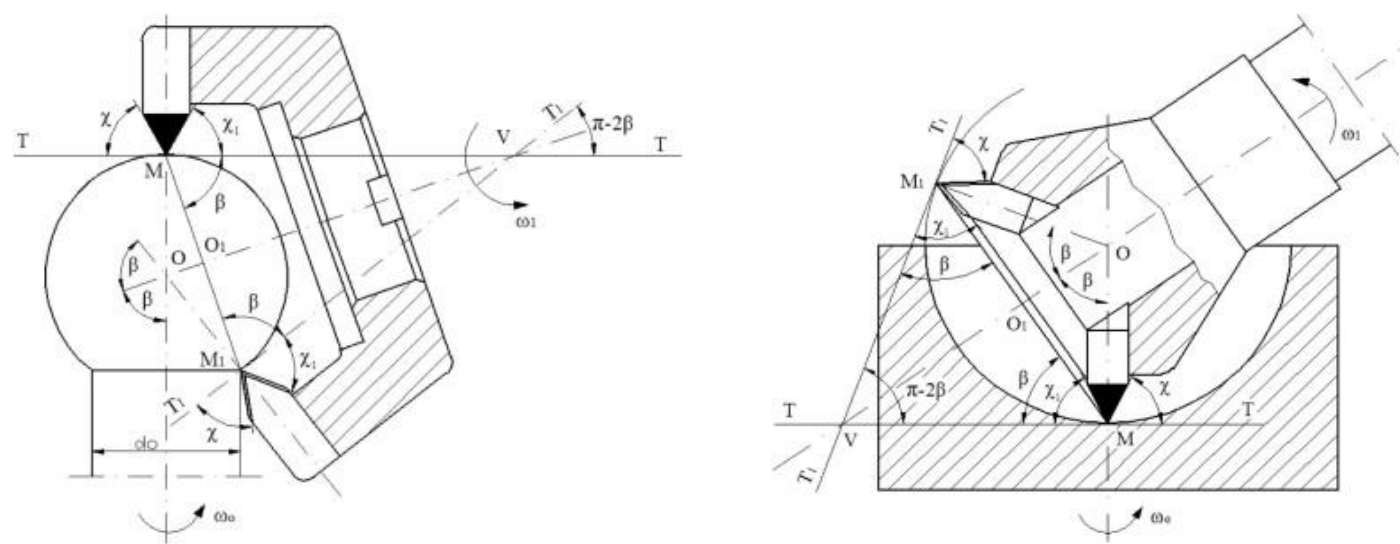

Fig. 6. Machining of spherical surfaces by milling with face insert milling cutters $[6,7]$

Figure 7 shows a particular example of milling head with face inserts, possibly usable also for machining spherical surfaces, protected by patent US 3315332 [8].

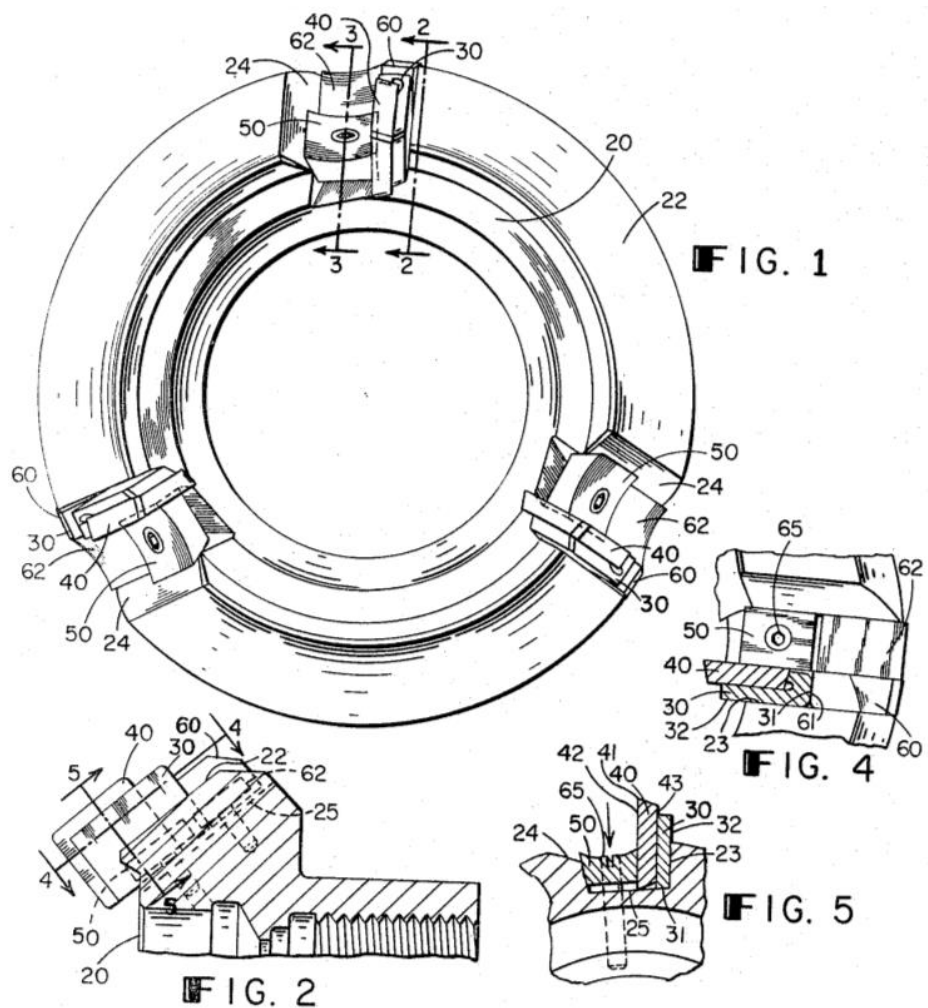

Fig. 7. Face insert milling cutter - a patented solution [8]

Obviously it is possible to machine spherical surfaces where the directrix and generatrix are programmed by means of NC milling machines and ball and mills, which are tools developed mainly for milling complex spatial surfaces. A large diversity of constructive solutions can be identified for this type of tools, many of which are patented $[3,9,10]$.

The conducted study highlights that:

- spherical surfaces can be machined efficiently by turning and milling, the preferred procedure being milling;

- most often more or less specific devices and tool holders are used;

- the utilisation of particular tools can be observed, most often using special design inserts.

In the particular case focussed on by the authors, eventually they opted for conceiving a turning device equipped with two profiled prismatic turning tools, easily sharpened by circular grinding. 


\section{Device for Turning Hemispherical Ends - the Constructive Solution}

The device - for that the number of considered constructive variants was narrowed down to two - is conceived to machine the hemispherical ends of the headrest support rods by the method of axial feed, using two profiled prismatic turning tools, thus having a materialised generatrix. The device can be mounted on a lathe, a drilling machine or even a milling machine. For any of these cases the focus was on enabling the device to carry out both the main rotation and the axial feed. On any machine-tool the machining procedure is turning.

Regardless of the constructive variant the device consists of a body, two profiled prismatic turning tools displayed radially (radially, eccentrically and tilted) and several components used mainly for adjusting tool position and clamping the tools. One of the constructive variants (figures 8 and 9) has a solid body 1 , the pockets $\mathrm{d}$ for the prismatic turning tools being machined in it by mortising or EDM.

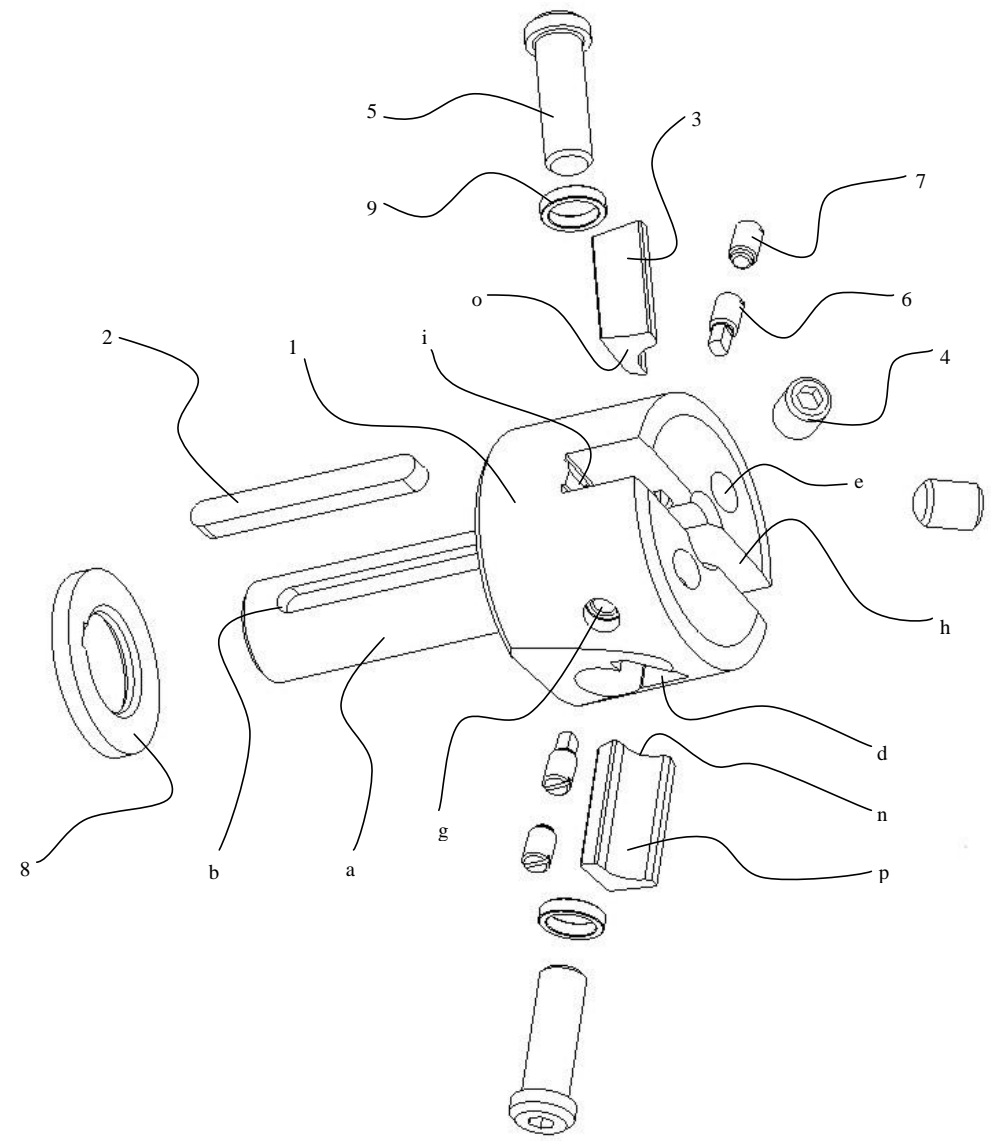

Fig. 8. Device for turning hemispherical ends, variant with a solid body. Exploded isometric view

The front face o, which is the conical end flank - obtained by circular milling - of the profiled prismatic turning tool 3 is supported by the spherical front face of the adjusting screws 6 , their axial position in the threaded holes g being blocked by screws 7. For any turning tool 3 the adequate contact of its front face with the front face of the corresponding adjusting screw 6 is ensured by means of a pushing screw 5 .

The position of screw 6 is adjusted by means of an adjusting plate 10 (figure 10). This is fixed by means of a screw 11 in the median groove of body 1 of the device, so that surface I of groove h comes into contact with the corresponding surface l of the adjusting plate 10. A profiled prismatic turning tool 3 is placed into the corresponding pocket, and then pushed by means of screw 5 until its front surface comes into contact with the plane surface $\mathrm{k}$ of adjusting plate 10. This position of turning tool 3 is clamped firmly by means of a clamping screw 4 that presses onto surface $p$ of turning tool 3 . The adjusting plate 3 is then removed and an adjusting screw 6 is screwed into the corresponding hole 6 
until its hemispherical front surface comes into contact with the rake face of turning tool 3 (figure 11). This position of the adjusting screw 6 is then blocked by means of a screw 7 . The adjusting plate 10 is removed and the process is repeated in order to adjust and block the position of the second adjusting screw 6.
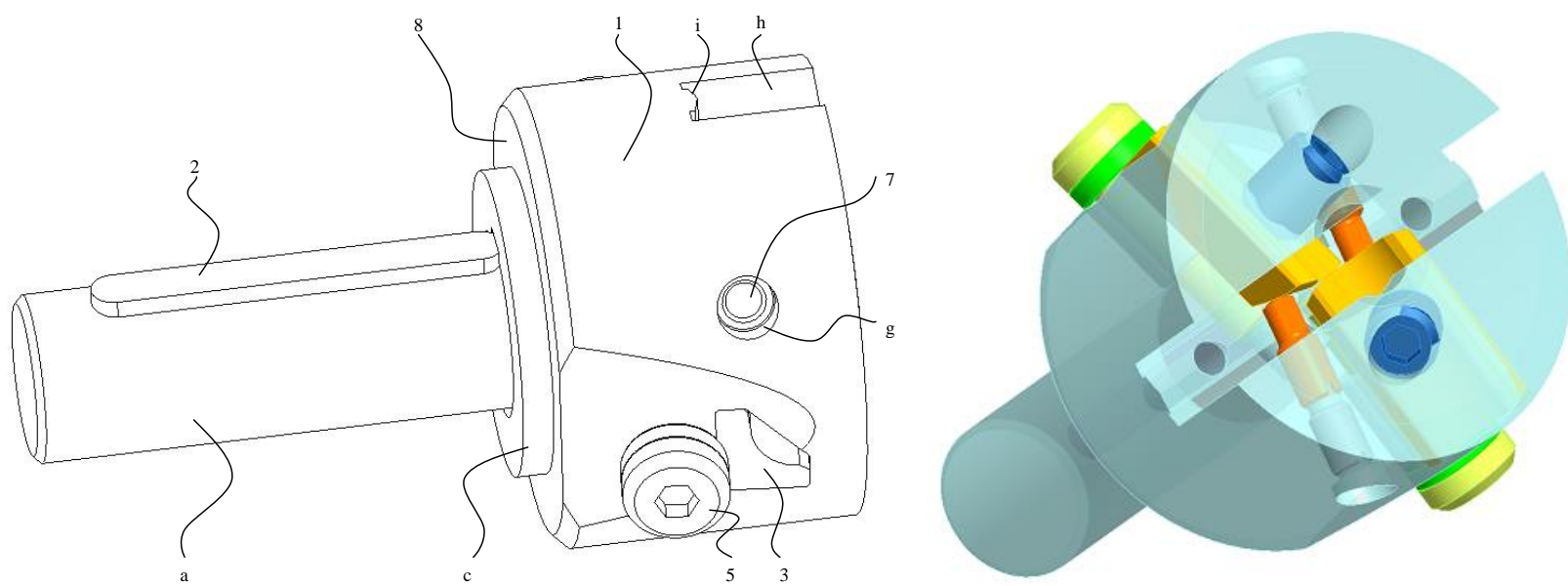

Fig. 9. Device for turning hemispherical ends - variant with a solid body. Isometric views
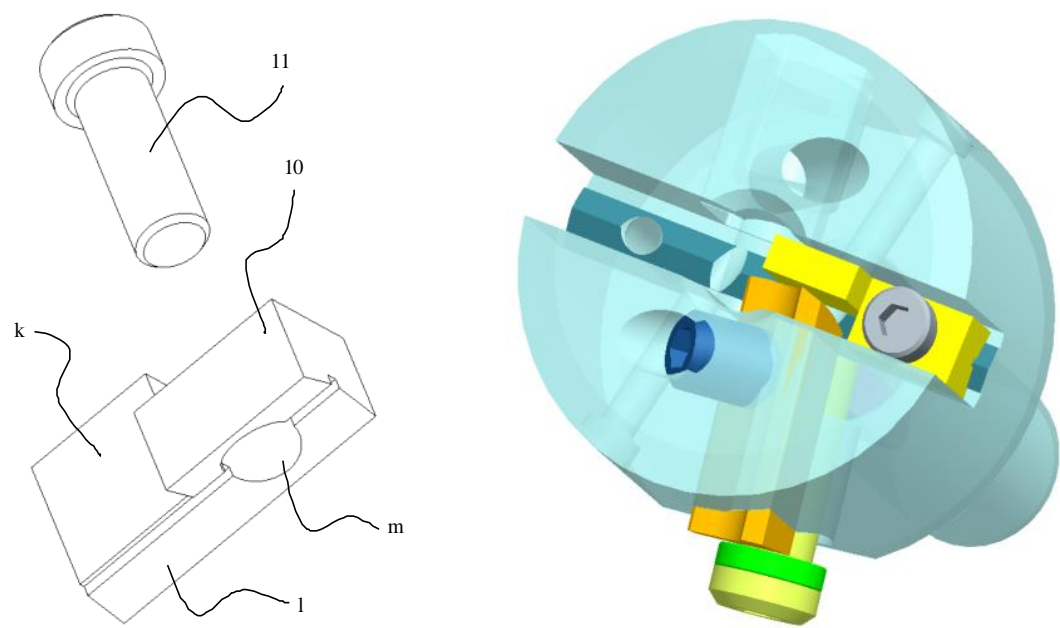

Fig. 10. Adjusting plate and its location in groove i of the device body
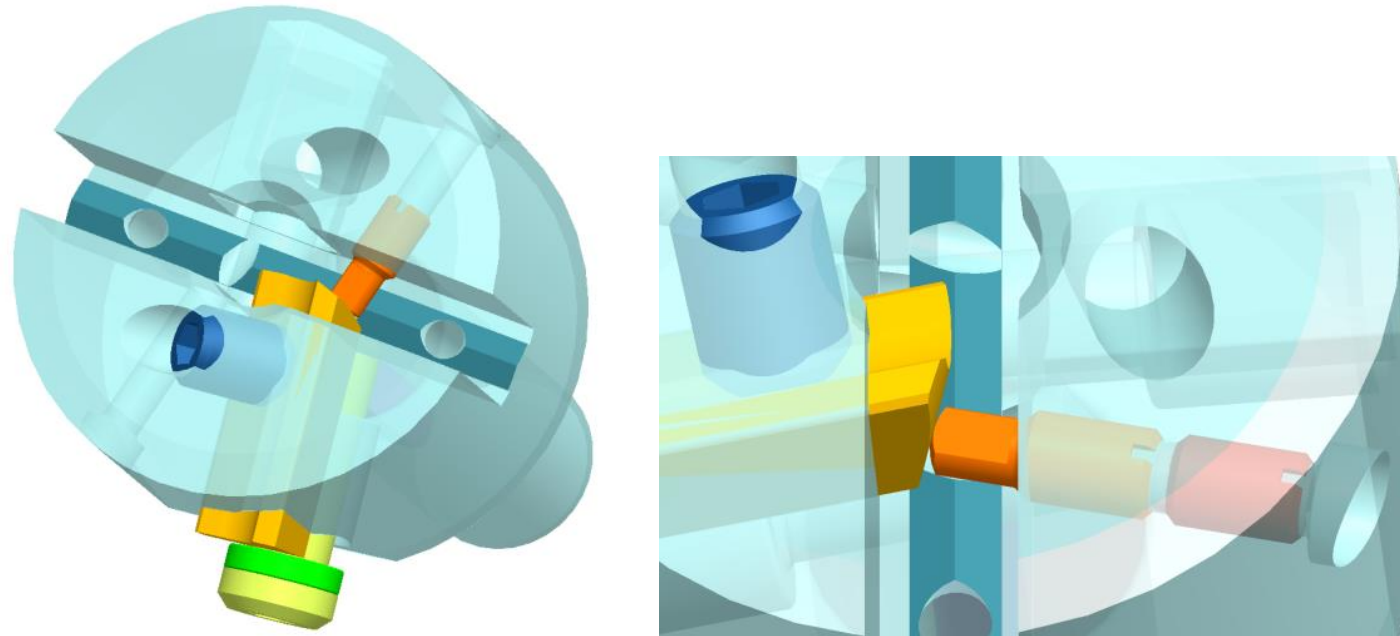

Fig. 11. Correct position of an adjusting screw 6 and its blocking modality 
Once the correct positions of the adjusting screws 6 and their subsequent blocking have been achieved, after any re-sharpening or replacement of the profiled prismatic turning tools 3 or their front faces o will come into contact every time with the front surfaces of the adjusting screws 6 and implicitly the cutting edges of $\mathrm{n}$ of the turning tools 3 will be in the correct positions in relation to the device.

The shaft a of the device can be cylindrical or conical, with or without a threaded hole depending on the machine selected for driving the tool. Specifically, the shape of the shaft is established depending on the geometry of the driving main shaft of the device. In figures 8, 9 and 10 the device has a cylindrical shaft, and is driven by means of a wedge.

If necessary the axial position of the device can be adjusted by a distancing element 8 of exactly defined length, figures 8 and 9.

In the other constructive variant, figures 12 and 13, the actual body 12 of the device is completed by two closing elements 13 , each being assembled to body 12 by means of two screws 17 , and which press onto the profiled prismatic turning tools 3 by means of a semi-cylinder 14 . This more complex constructive solution of the device was adopted for technological reasons, in order to allow the accurate milling of the plane supporting surfaces in the pockets $r$, where the profiled prismatic turning tools are placed, figure 14 .

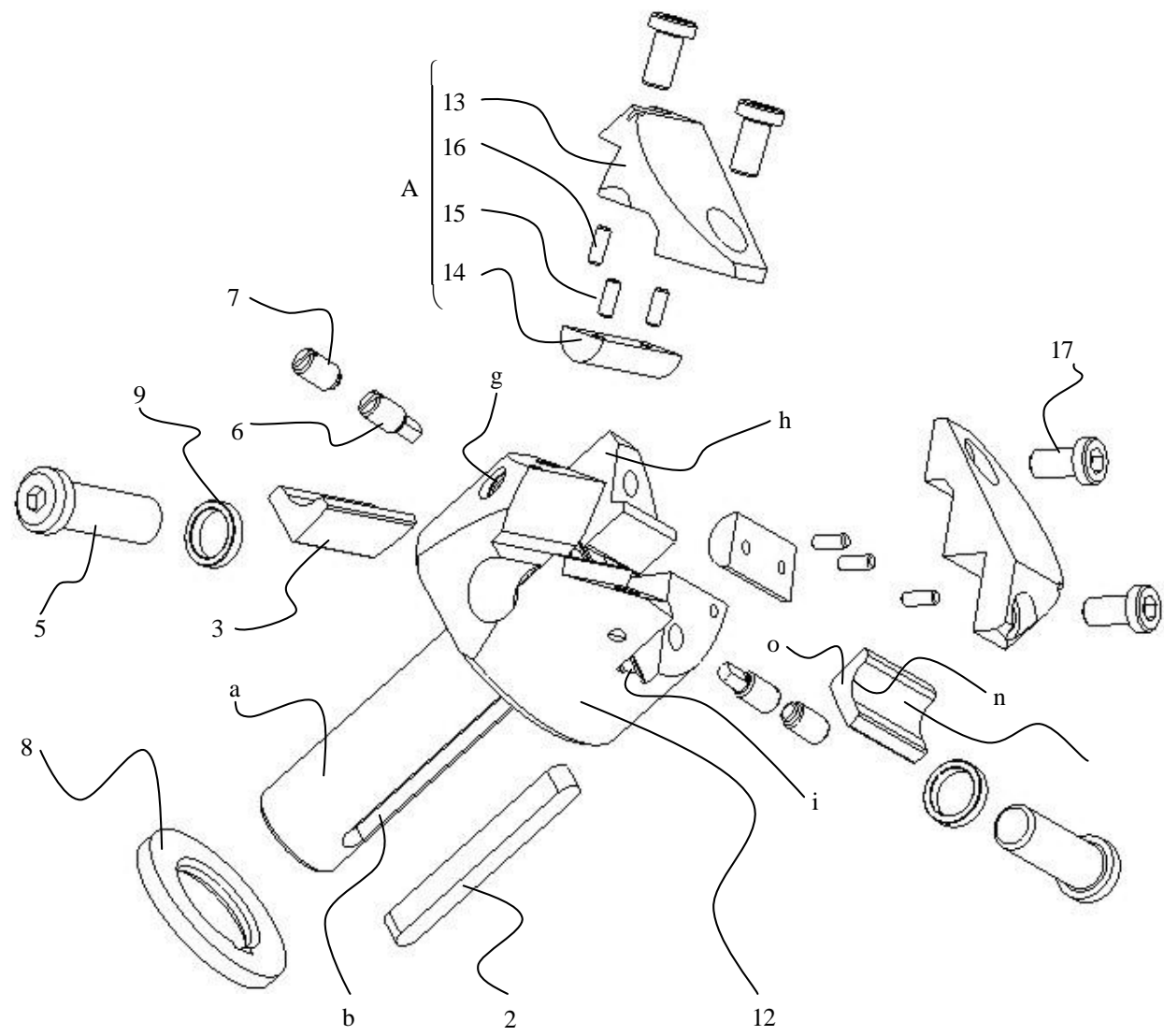

Fig. 12. Device for turning hemispherical ends, variant with a milled body. Exploded isometric view

The identified benefits of the device include:

- easy deploying;

- compactness and constructive simplicity;

- a single initial adjustment of the turning tool positions is sufficient;

- repeatability of turning tool repositioning after each re-sharpening;

- simultaneous machining of both headrest support rod hemispherical ends is possible by using two identical devices in parallel;

- increased productivity; 
- device usable on various machine-tools - lathes, drilling machines or even milling machines universal or not.
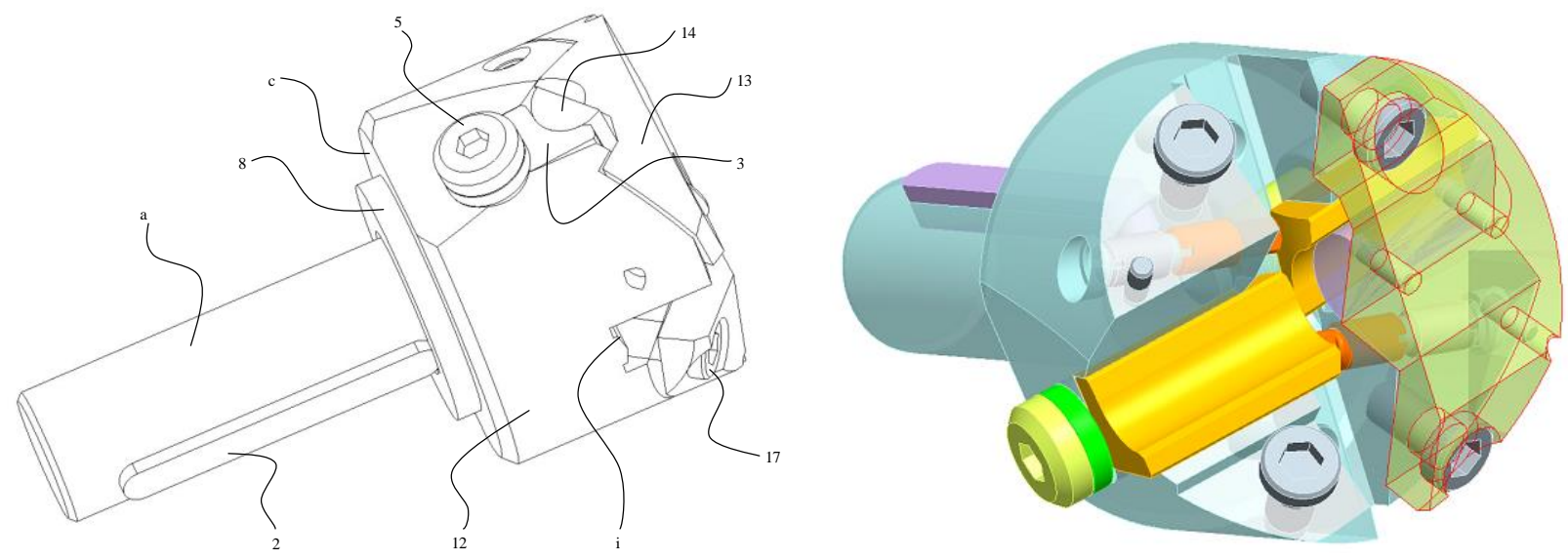

Fig. 13. Device for turning hemispherical ends, prototype. Isometric views

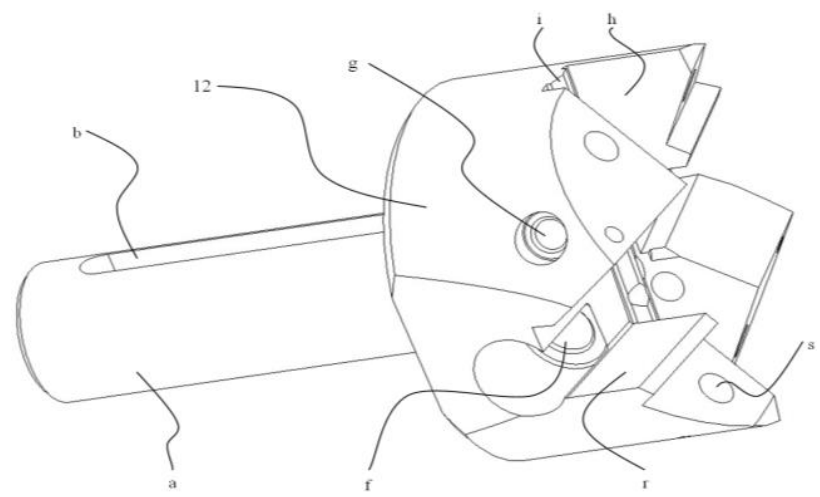

Fig. 14. Milled body of the device, isometric view

For both constructive variants of the device invention patent protection was requested and obtained [11]. The originality of the solutions was recognised and appreciated at national and international inventics fairs; the patent obtained several prizes, medals and diplomas, including a gold medal in one of the International Exhibitions of Inventions of Geneva, Switzerland.

\section{Conclusions}

Machines manufacturing stands for a wide range of components characterised by various combinations of surfaces; the most common ones being plane, cylindrical and even conical. Apart from roller bearing elements, components featuring spherical surfaces are infrequent.

Customized technologies are deployed for the machining of components with less common surfaces, technologies requiring either specialised/special machines or specific tools and/or devices or both. Even though it is not an imperative requirement, conceiving and using custom tools is a relatively frequent decision that while entailing a relatively small investment always contributes to increasing productivity. In many cases the preferred solution is to use a more or less specialised device on the available machine-tools. This is more cost intensive than a custom tool, but yields a significant increase of productivity. Often one or more adequate tools are needed as well.

A particular case identified by the authors is the headrest support rod with hemispherical ends encountered in certain motor vehicles. This is a mass-produced component. The hemispherical ends can be machined either prior to or after the bending of the support, in which latter case machining of the hemispherical ends becomes difficult because the support needs to be set into rotation.

In literature efficient modalities for the generation of hollow and concave spherical surfaces are 
presented, and tools and devices dedicated to the machining of spherical surfaces are identified. Frequently ball end mills are encountered using inserts or not, however not dedicated to the machining of hollow surfaces.

Upon having studied the machining possibilities of the headrest support rod hemispherical ends the team of authors considered as efficient the utilisation of a very specific device that allows the machining of the hemispherical ends subsequently to the bending of the support, the motions required for the machining being carried out by the device. Also considered was the possibility of using two identical devices in parallel for the simultaneous machining of both headrest support ends.

The device designed in two constructive variants is conceived for the machining of the hemispherical ends of the headrest support rod by the method of axil feed using profiled prismatic turning tools, thus with a materialised generatrix. The device can be used on a lathe, a drilling machine or even a milling machine. In all of these cases the priority desideratum was to develop a device that carries out both the main rotation and the axial feed. Regardless of the utilised machine-tools the machining procedure is turning.

In one of the constructive variants the body of the device is solid and the pockets for the profiled prismatic turning tools are machined by mortising or EDM. The second more complex constructive variant was adopted for technological reasons in order to allow accurate milling of the plane support surfaces of the pockets for the profiled prismatic turning tools.

In both constructive variants of the device invention patents were requested and obtained.

The originality of the solutions was recognised and appreciated at national and international inventics fairs; the patent obtained several prizes, medals and diplomas, including a gold medal in one of the International Exhibitions of Inventions of Geneva, Switzerland.

\section{Acknowledgment}

I dedicate this paper to the memory of my brother, Eng. CIOARĂ Silviu Constantin, who initiated this research and contributed decisively to the conception of the device.

\section{References}

1. https://i.ytimg.com/vi/sH7NNT8hLpc/maxresdefault.jpg

2. Dier S.A.: Apparatus for machining a spherical surface. Patent US 3142117

3. Allaire P.B., Stashko D.R.: Cutting tool and insert therefore. Patent US 4618296

4. Kress D., Strom R.: Tool for chipping a concave, spherical surface. Patent EP 1458511

5. Smith R.J.: Method for cutting internal spherical surfaces. Patent US 3212405

6. https://ars.els-cdn.com/content/image/1-s2.0-S0307904X12007809-gr7.jpg

7. https://ars.els-cdn.com/content/image/1-s2.0-S0307904X12007809-gr8.jpg

8. Lowry R.D., Strout R.B.: Milling cutter. Patent US 3315332

9. Lindberg H., Wallström L.-G.: Ball end mill and throw away insert for such end mill. Patent EP 0462954

10. Mizuno T., Yoneyama S.: Throw away insert and end mill. Patent US 4252480

11. Tîțu M.A., Oprean C., Cioară S.C., Cioară Gh.R., Durdun E. Rachieru N. Sabău D.: Dispozitiv pentru strunjit capete semisferice (Device for lathe-turning of semi-spherical ends). Patent R0 129632 B1 